\title{
Methodische Probleme bei der Suche nach Strukturunterschieden in einem städtischen Raum: Detaillierte Auflösung versus Regionalisierung
}

\section{Einleitung}

Wichtiger Impetus für diese Arbeit war das schwelende Unbehagen über die verbreitete Verwendung (Publikation von BFS, kantonalen statistischen Ämtern, NFP-25) von gemeindeweise aufgeschlüsselten Daten für die Deskription und Analyse von räumlichen Strukturunterschieden in städtischen Regionen. Da die Bevölkerungszahlen der administrativen Einheiten in einem städtischen Raum üblicherweise wesentlich stärker variieren als in eher ländlichen Gebieten, decken solche Darstellungen häufig mehr zufällige als relevante Unterschiede auf. Wegen der unterschiedlichen Informationsdichte sind Analysen auf Gemeindeebene für die meisten Fragestellungen ungenügend. Wenn zudem, was bei den meisten Choroplethenkarten leider üblich ist, das ganze Gemeindegebiet mit derselben Farbe oder demselben Grauwert eingefärbt wird, werden die Verzerrungen zugunsten flächengroßer, relativ schwach besiedelter $\mathrm{Ge}$ biete noch größer. Die teilweise beträchtliche Heterogenität innerhalb der Gemeindegrenzen kommt dagegen nicht zum Ausdruck, schon gar nicht, wenn - was bedauerlicherweise ebenfalls häufig vorkommt - nicht einmal die bevölkerungsreichen Städte weiter aufgegliedert werden. Außerdem verlaufen die Gemeindegrenzen in städtischen Räumen nicht selten mitten durch das Siedlungsgebiet, weshalb einzelne Ortsteile auf das Zentrum der Nachbargemeinde und nicht auf das u. U. weiter entfernte Zentrum ihrer eigenen Gemeinde ausgerichtet sind.

Die Zufälligkeit von Orts- und Ortsteilgrenzen und ihre Nichtübereinstimmung mit den funktionalen Grenzen ist ein grundsätzliches Problem, auch wenn die von CAROL (1956:89) geforderte Zusammenlegung von Gemeinden und Bezirken («überholte Einheiten mittelalterlicher Provenienz») in der Schweiz zumindest im Umkreis der Städte kaum mehr ein Thema ist. Da die Grenzen der Stadtgemeinden schon lange nicht mehr mit den funktionalen Grenzen der Städte übereinstimmen, sind statistische Auswertungen und kartographische Darstellungen, die das Umland nicht einbeziehen, unvollständig, wenn nicht sogar irreführend.

Es soll im folgenden versucht werden, am Fallbeispiel Zürich Grundlagen für ein zutreffenderes Bild eines städtischen Raumes, als es eine Darstellung auf Gemeindeebene erlaubt, abzuleiten. Dabei bieten sich zwei Möglichkeiten an: die Zusammenfassung zu sinnvollen Teilregionen sowie die Verwendung von möglichst kleinräumig aufgeschlüsselten Daten.

\section{Modelle zur Gliederung städtischer Räume}

Regionalisierungen auf verschiedenen Ebenen gehören zu den traditionellen Aufgaben der Geographie (HAGGETT 1975). Entsprechend reich ist die Literatur über Regionalisierungsverfahren (z. B. SEDLACEK 1978, HAGGETT 1973). Die Regionalisierung ist sowohl Erklärungsansatz als auch Forschungsverfahren (HARVEY 1979). Vom Konzept her ist sie nichts anderes als eine spezielle Form der Klassenbildung (GRIGG 1970). Klassifikation wie Regionalisierung bezwecken die Ordnung der Forschungsobjekte; Ziel ist nicht die Einordnung von Objekten in Klassen per se, sondern die Möglichkeit von induktiven Verallgemeinerungen, die zum Verständnis des in Frage stehenden Gebietes oder des Untersuchungsgegenstandes beitragen (GRIGG 1970); dabei besteht eine Abhängigkeit von den erreichbaren Statistiken, die Kompromisse bei der Abgrenzung erfordert.

Die Literatur über mögliche Definitionen und Abgrenzungen von Stadtregionen ist beträchtlich (vgl. SCHULER 1984, BOPP 1991). Hingegen existieren so gut wie keine Arbeiten über mögliche innere Unterteilungen.

Die Gliederung des Stadtgebietes in konzentrische Gürtel (BURGESS 1925) bzw. in keilförmige Sektoren (HOYT 1939) gehört zu den ältesten und populärsten Modellen in der Stadtgeographie.' Auch wenn die Grundannahme eines freien Bodenmarktes in einem weitgehend überbauten und diversen Zonierungsvorschriften unterliegenden städtischen Gebiet fraglich ist, ermöglichen die beiden Modelle zusammen eine prägnante Abbildung von Ringelementen sowie von mono- und polyzentrischen Einflüssen, wie sie gerade in einem städtischen Raum häufig sind (NEBEN 1985).

Obwohl Gürtel- und Sektorkonzeption auch heute noch fruchtbare Ansätze für die Beschreibung von Strukturunterschieden in einem städtischen Raum liefern können, sind konkrete Umsetzungen selten (eine Ausnahme findet sich in LEY 1983:214).

Nachdem sich zwei solche Modelle in einer breiteren Anwendung bewährt haben (BOPP 1991), soll ihr Nutzen hier demonstriert werden.

Matthias Bopp, Dr. phil. II, Geograph,

Institut für Sozial- und Präventivmedizin

der Universität Zürich, Sumatrastr. 30, 8006 Zürich 


\section{Umsetzung auf die Agglomeration Zürich}

\section{Untersuchungsgebiet}

Als beste Annäherung an den funktionalen und den ökonomischen Stadtbegriff bietet sich die Agglomeration an, auch wenn die Stadt als gebaute Form nịcht mit Urbanität als Lebensstil gleichgesetzt werden darf, weil sie in Realität längst nicht mehr übereinstimmen (HARVEY 1973:307). Obwohl die Ausdehnung einer Agglomeration nur diffus wahrgenommen wird und die politische Bedeutung eher gering ist, ist der Begriff dennoch fest verankert im Bewußtsein der Bevölkerung (SCHULER 1984), und Lebens- sowie Konsumgewohnheiten sind stark auf das Zentrum ausgerichtet (BAJKA 1967). Seit über 100 Jahren werden in der Schweiz jeweils im Anschluß an die Volkszählungen Agglomerationen abgegrenzt. «Der Agglomerationsbegriff ... ist damit der einzige dynamische Regionalisierungsansatz, der in der Schweiz offiziell bestimmt und verwendet wird" (SCHULER 1984:25). Für die folgenden Beispiele dient die Agglomeration Zürich gemäß der Definition 1980 als Untersuchungsgebiet (SCHULER 1984; BOPP 1991; Abb. 1).

\section{Kleinräumige Aufgliederung}

In der Schweiz existieren unter dem Niveau Gemeinde keine vergleichbaren kleinräumigen Unterteilungen. Die Zählkreise der Volkszählungen werden nicht nach Homogenitätskriterien, sondern willkürlich festgelegt (wichtigstes Kriterium: "von einem Zähler zu bewältigen, d. h. etwa 100-150 Haushaltungen"). Sie haben jeweils nur für einen Zensus Gültigkeit und lassen meist keine kartographische Umsetzung zu. Die 81 Gemeinden der Agglomeration Zürich weisen bezüglich der Bevölkerungszahlen sehr unterschiedliche Größenordnungen auf. Das Verhältnis der Einwohnerzahlen zwischen der größten (Stadt Zürich) und der kleinsten Gemeinde (Islisberg) betrug bei der Volkszählung 1980 1:2350; mit der amtlichen Einteilung der Stadt Zürich in 34 Quartiere - die meisten davon aus früher selbständigen Gemeinden hervorgegangen - läßt sich dieses Verhältnis auf rund 1:175 reduzieren (114 Einheiten).

In der Stadt Zürich besteht seit einigen Jahrzehnten eine Aufgliederung des Stadtgebietes in 202 statistische Zonen mit meist zwischen 500 und 3000 Einwohnern. Die Grenzen folgen im Siedlungsgebiet i. a. größeren StraBenzügen, ohne jedoch den Detaillierungsgrad einer Baublockstatistik zu erreichen. Die Homogenität innerhalb der Zonen wird nur teilweise gewahrt. Für die übrigen Agglomerationsgemeinden konnte die mit der Volkszählung durchgeführte Siedlungsstatistik des Kantons Zürich nutzbar gemacht werden, wozu einige Anpassungen nötig waren (BOPP/HERZOG 1987; BOPP 1991: 16 ff.). Von 49 Zürcher Agglomerationsgemeinden (ohne Stadt Zürich), die Siedlungen mit über 2000 Einwohnern aufwiesen, haben 33 eine Unterteilung in Quartiere durchgeführt. Einige dieser Quartiere sind etwas groß ausgefallen (acht zählten 1980 über 4000 Einwohner). Für sieben Gemeinden ohne Quartiereinteilung, aber mit hektarweiser Auszählung der Volkszählung 1980 (Bachenbülach, Bassersdorf, Buchs, Dietikon, Dietlikon, Oberengstringen, Zollikon), konnten die Zählkreise rekonstruiert und 37 Quartiere nach dem Homogenitätskriterium gebildet werden. Nur sieben Gemeinden mit über 3000 Einwohnern (bzw. nur eine mit über 5000 Einwohnern) konnten nicht unterteilt werden.

Für die kartographische Aufbereitung wurde eine Reduktion der ausgeschiedenen Einheiten auf das effektive Siedlungsgebiet durchgeführt; ohne größere Bauabstände aneinander angrenzende Kleinsiedlungen wurden fusioniert. Alle Siedlungseinheiten mit mindestens 50 Einwohnern in Privathaushalten und einer minimalen Siedlungsfläche von 1 ha wurden digitalisiert, falls sie nicht mehrheitlich aus Industrieareal bestanden. Nach diesen Arbeiten blieben 754 Einheiten übrig, 85\% davon mit einer Einwohnerzahl zwischen 100 und 3500; das Verhältnis zwischen der Einheit mit den wenigsten und derjenigen mit den meisten Einwohnern beträgt 1:140 (BOPP 1991:18). Diese 754 Einheiten konnten mit den Individualdaten der Eidgenössischen Volkszählung 1980 gekoppelt werden. Auch wenn diese mittlerweile etwas an Aktualität eingebüßt haben, sind sie dank ihres Detaillierungsgrades für viele Fragestellungen von unschätzbarem Wert, zumal der Fragebogen für die Volkszählung 1990 gegenüber 1980 stark reduziert wurde.

\section{Gürtelmodell}

Für das Gürtelmodell wurden die Agglomerationsgemeinden und die Zürcher Stadtquartiere nach dem Zeitpunkt des Einbezugs in den Verstädterungsprozeß klassiert. Im Raum Zürich bieten sich dafür die beiden städtischen Eingemeindungen von 1893 und 1934 sowie die Agglomerationsabgrenzungen gemäß den Volkszählungen 1950, 1960, 1970 und 1980 an. ${ }^{2}$ Das 1893 eingemeindete Gebiet wurde in einen inneren und äußeren Gürtel aufgeteilt, wobei die Trennlinie etwa der Siedlungsgrenze um 1920 folgt. Insgesamt ergeben sich dadurch neben dem Kern - der ursprünglichen Stadtgemeinde Zürich (heute Stadtkreis 1) - sieben Gürtel; durch die Umsetzung dieser acht Zonen in ein bevölkerungsproportionales Kartogramm können bevölkerungsbezogene Variablen ihrem wahren Gewicht entsprechend abgebildet werden (Abb. 2).

\section{Sektorzonenmodell}

Das Sektorzonenmodell orientiert sich an den Verkehrsachsen. Fast alle Städte haben sich vom historischen Zentrum entlang den Verkehrsachsen nach außen entwickelt. Erst in späteren Phasen - oft erst mit der raschen Verbreitung des Autobesitzes seit den 50er Jahren - wuchsen die zunächst unbebauten Keile zwischen den Verkehrsachsen zu (PFEIL 1972). Hinter dem Sektorzonenmodell steckt die These einer Fortschreibung innerstädtischer 


\section{Die Gemeinden der Agglomeration Zürich}

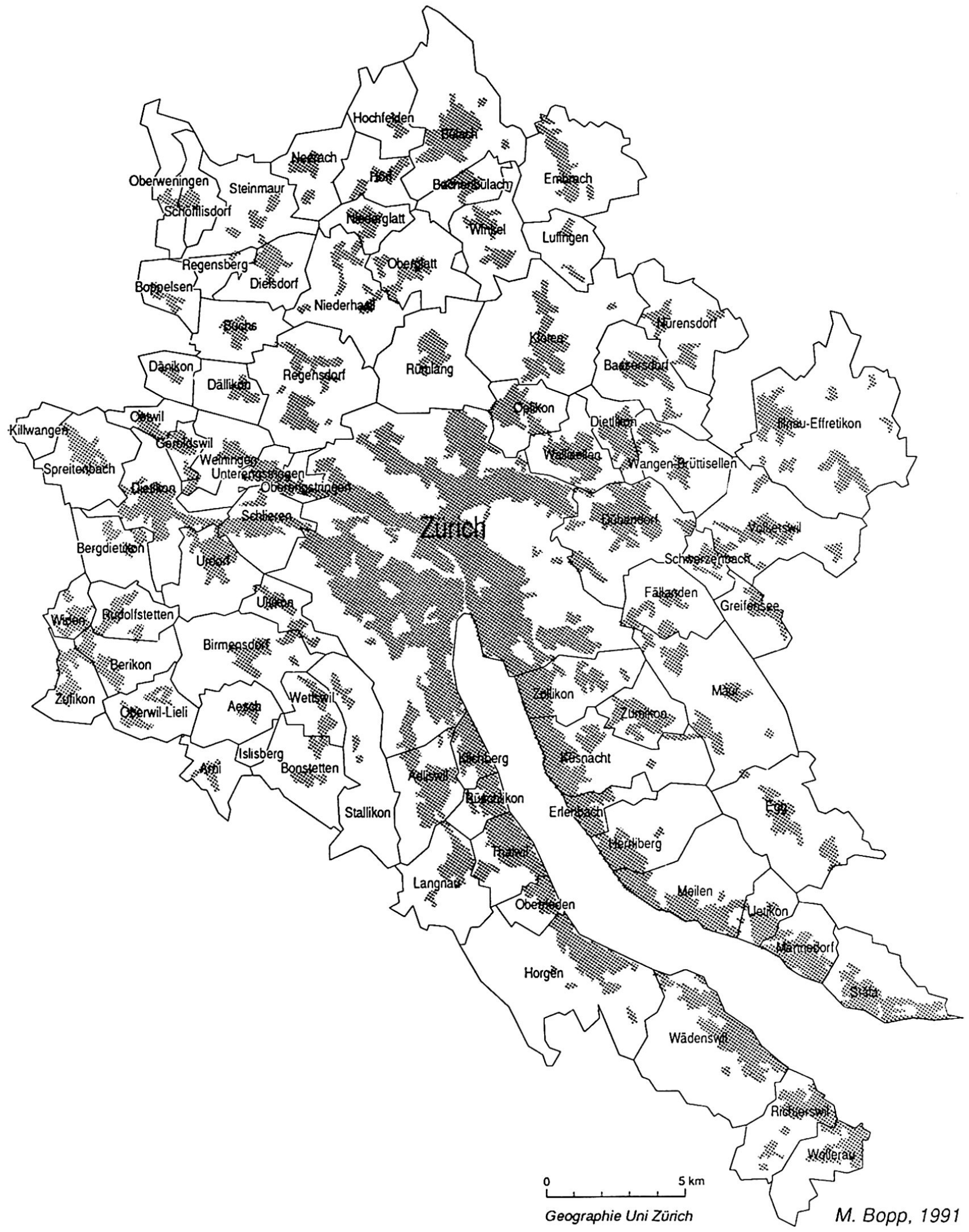

Abb. 1 Die Gemeinden der Agglomeration Zürich in der Definition Volkszählung 1980. 
Entmischungsmuster in die anschließenden Agglomerationsachsen (GÜLLER et al. 1980). Eine starke Bindung an die einmal gewählte Achse wurde für Migration und Pendlerbeziehungen aufgezeigt (GULLER et al. 1980; ROHNER 1985). Es erstaunt daher nicht, daß die Segregration nach Achsen in vielen Schweizer Agglomerationen ausgeprägt ist, die Segregrationstendenzen scheinen sich sogar noch zu verstärken (SCHULER 1984). In der Agglomeration Zürich sind die meisten Achsen durch die Topographie vorgegeben; einzig am Nord- und Ostrand sowie bei der Unterteilung der Achsen besteht ein gewisser Spielraum. Die hier gewählte Einteilung in 27 Sektorzonen berücksichtigt nach Möglichkeit die Gemeindegrenzen; wie beim Gürtelschema wurde aus dieser Gliederung ein bevölkerungsproportionales Kartogramm abgeleitet (vgl. BOPP 1991:19 ff.; Abb. 3).

\section{Bewertung}

Das am Geographischen Institut der Universität Zürich entwickelte Kartographiesoftwarepaket PSCOPAM (HERZOG 1988) garantiert dank seiner stufenlosen Wertabbildung eine adäquate Abbildung von großen und kleinen Unterschieden in den Datenwerten. Damit wird eine gleichzeitige Darstellung derselben Variable auf verschiedenen Aggregationsniveaus möglich. ${ }^{3}$ Die detaillierte Karte kann daher mit den zusammenfassenden Kartogrammen kombiniert werden, wie hier am Beispiel des Anteils der Direktoren und leitenden Angestellten (Abb. 4).

Die Überlegenheit gegenüber einer Gemeindekarte ist offenkundig: Die beiden Kartogramme erlauben eine rasche Übersicht (Gürtelschema: relativ weniger Direkto-

\section{Gürtel-Schema der Agglomeration Zürich}

unten: flächentreue Darstellung

rechts: Kartogramm (Flächen proportional zu den Bevölkerungszahlen 1980)

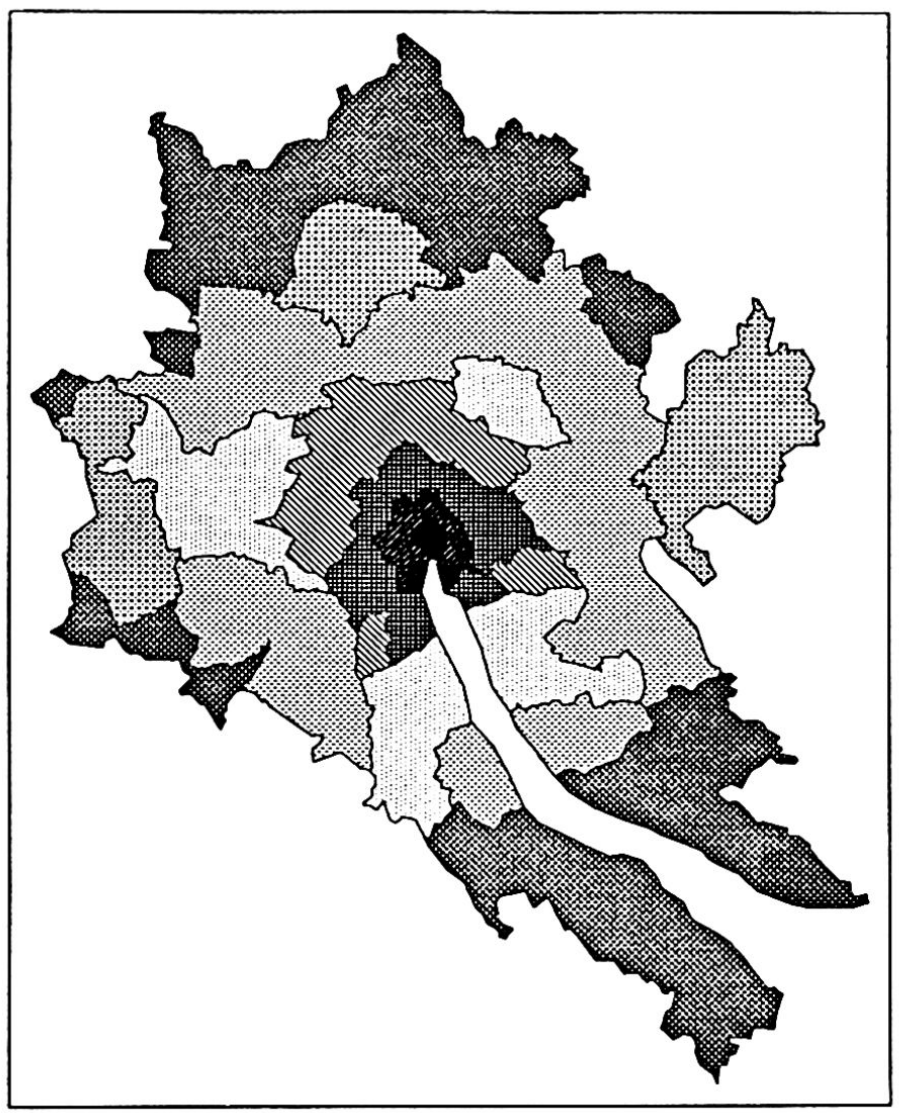

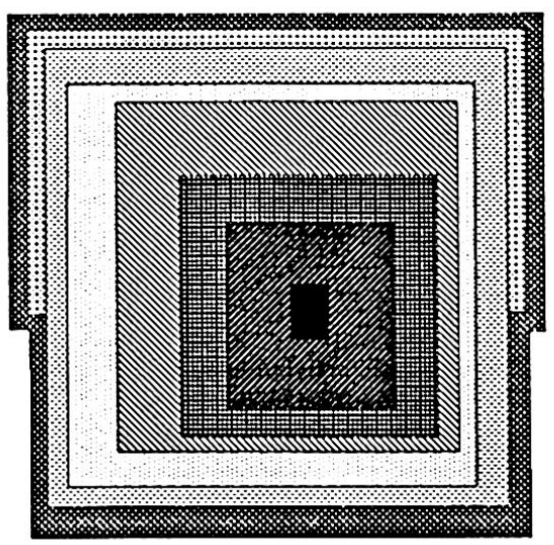

Stadt Zürich bis 1892

1892 eingemeindet, heute im CityEinflussbereich

1892 eingemeindet, nicht im CityEinflussbereich

1934 eingemeindet

\section{Agglomerationsgemeinden} 1950

zusätzliche Gemeinden

1960

zusätzliche Gemeinden

1970

zusätzliche Gemeinden

1980 


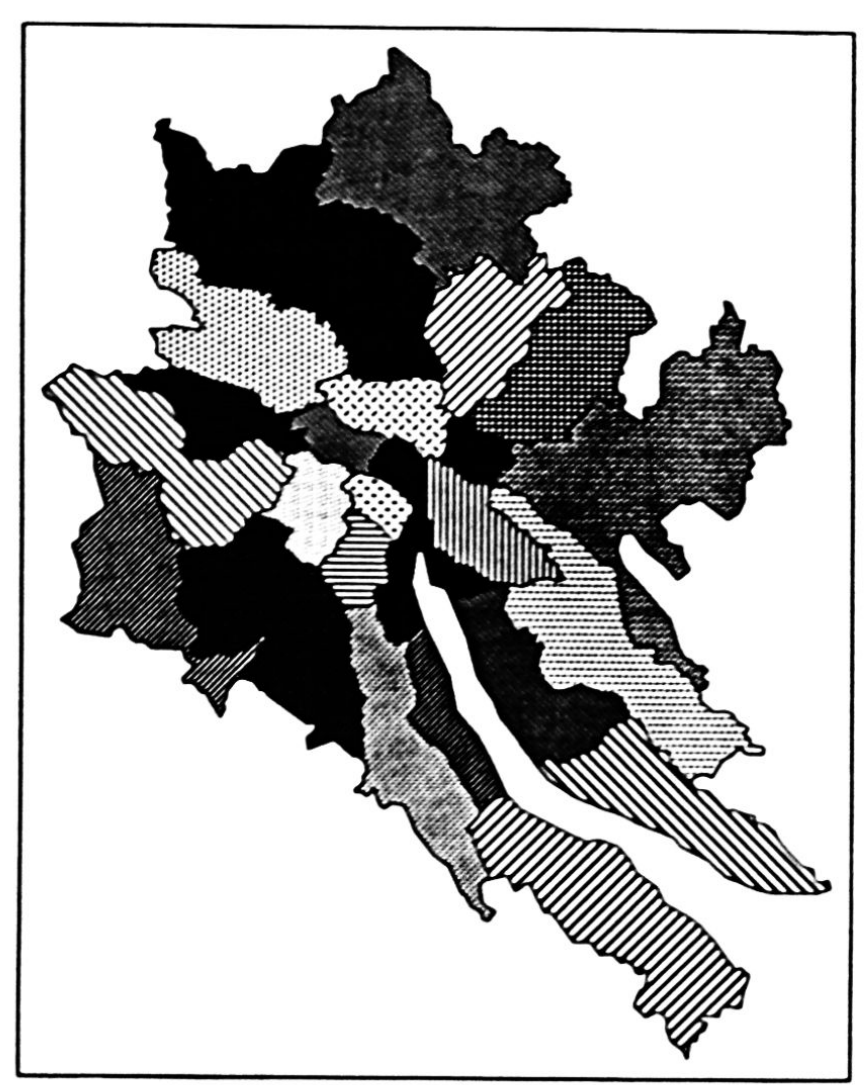

\section{Sektorzonen-Schema der Agglomeration Zürich}

links: flächentreue Darstellung

unten: Kartogramm (Flächen proportional zu den Bevölkerungszahlen 1980)
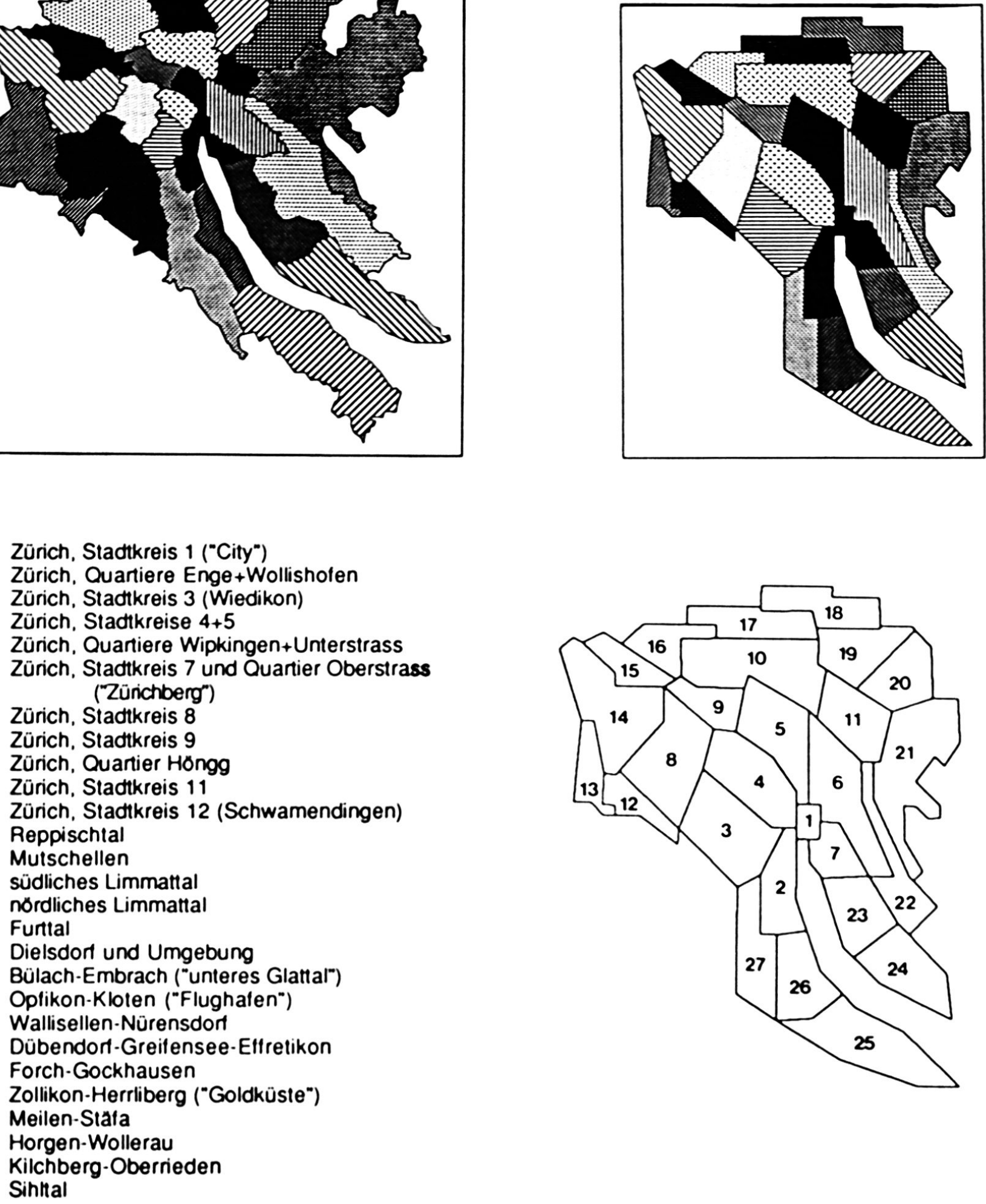


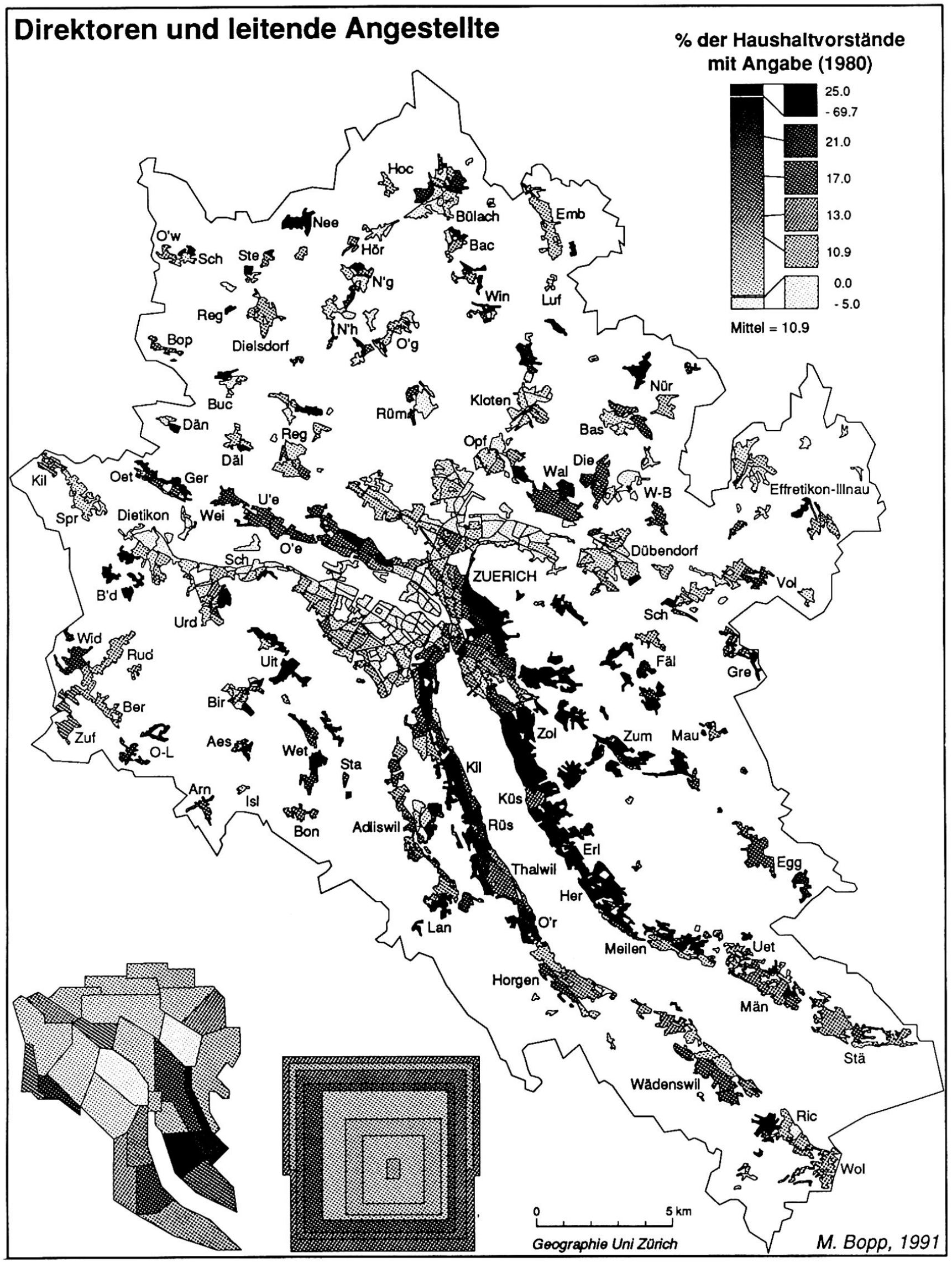

Abb. 4 Direktoren und leitende Angestellte in der Agglomeration Zürich (Grundlage: Individualdaten der Volkszählung 1980). 
ren und leitende Angestellte in der Stadt Zürich als im übrigen Agglomerationsgebiet, Maximum im ersten Vorortgürtel, sekundäres Minimum im dritten. Sektorzonenschema: hohe Werte an rechtem Zürichseeufer ["Goldküste»], Pfannenstiel, Zürichberg, stadtnahem linkem Seeufer und südwestlich der Stadt Zürich, sekundäre Maxima in den Achsen Höngg-Oetwil und Wallisellen-Nürensdorf sowie im Mutschellen-Gebiet, Tiefstwerte in den Zürcher Stadtkreisen 4/5 und 12, generell tiefe Werte in fast der ganzen nördlichen Hälfte der Agglomeration und auf der Südseite des Limmattals). In der detaillierten Darstellung treten markante lokale Disparitäten auf, die bei einer gemeindeweisen Betrachtung unerkannt geblieben wären (besonders deutlich in Langnau, Richterswil, Nürensdorf und Urdorf). Die sozialräumliche Terrassierung des Zürichbergs wäre bei einer Darstellung nach Stadtquartieren ebenfalls nicht zum Ausdruck gekommen. Damit ist erst ein geringer Teil der Information ausgeschöpft, die ein mit den örtlichen Verhältnissen vertrauter Karteninterpret aus dieser Abbildung zu ziehen vermag.

Das Sektorzonenmodell ist für die Wiedergabe von Segregationserscheinungen (Haushaltstruktur, Einkommen, Sozialstatus) besonders geeignet, das Gürtelmodell dagegen für Stadt-Land-Unterschiede (Bevölkerungsdichte, Überalterung, Motorisierungsgrad, Angebot an Läden und Dienstleistungen) meist überlegen (BOPP 1991).

\section{Aneinanderreihung von gleichartigen Darstellungen}

Gürtel- und Sektorzonenmodell ermöglichen meist eine prägnantere Sicht der Strukturunterschiede, was eine Generalisierung erleichtert. Die Nützlichkeit der Aneinanderreihung von kartographischen Darstellungen für eine Zusammenschau mehrerer zum gleichen Kontext gehörender Variablen wurde schon mehrfach demonstriert (BERTIN 1974). Für diesen Zweck eignen sich Gürtel- und Sektorenmodell ausgezeichnet. Daneben können sie auch für einen Überblick über Entwicklungstrends verwendet werden. Als Beispiel diene die Bevölkerungsentwicklung von 1920 bis 1990 (Abb. 5 und 6). Das Gürtelschema belegt nicht nur die Wachstumsspitzen in den 40er, 50er und 60er Jahren, sondern demonstriert auch, wie sich die Zone des größten Wachstums kontinuierlich vom Stadtkern wegbewegt hat. Der 4. Gürtel (Außenquartiere der Stadt Zürich), während Jahrzehnten die Zone mit dem stärksten Bevölkerungswachstum, unterscheidet sich seit 1960 nur noch geringfügig von den inneren Gürteln. Im Sektorzonenschema wird deutlich, daß die nördliche Agglomerationshälfte durchwegs höhere Wachstumsraten hatte als die südliche. Seit 1980 sind die Unterschiede zwischen den Sektorzonen gering: Nur noch vereinzelte Randzonen zeigen ein nennenswertes Bevölkerungswachstum; in der Stadt Zürich sind die Bevölkerungsabnahmen in den citynahen Gebieten fast zum Stillstand gekommen.

\section{Aussagekraft der Modelle im Vergleich}

Wie alle deskriptiven Methoden können die oben vorgestellten Modelle keine Kausalitäten belegen, sondern bloß Hinweise auf mögliche Einflußfaktoren geben (Gefahr der ecological fallacy, vgl. BAHRENBERG/GIESE 1975:198 f.). Von Vorteil ist die Ableitung der Regionen aus Topographie und historischer Entwicklung, also nicht aus einem statistischen Gruppierungsverfahren unter Verwendung von Volkszählungsdaten. Die beiden Regionalisierungen können daher ohne die Gefahr der Tautologie für die Interpretation von Volkszählungsdaten verwendet werden.

Der Vorgang der Aggregation ist in der Regel mit einem Informationsverlust verbunden, der in der Abnahme der Streuung seinen statistischen Ausdruck findet. Diesem Verlust steht ein Gewinn an Übersichtlichkeit und Ordnung gegenüber. Der Vergleich der Verteilungen von Merkmalen erfordert ein Maß, das unabhängig von der Dimension der Merkmale und der Zahl der Beobachtungseinheiten ist. Ein solches Maß ist der Variationskoeffizient von PEARSON (Standardabweichung in Prozenten des arithmetischen Mittels). Anhand verschiedener Variablen sollen Gürtel- und Sektorzonenmodell miteinander sowie mit der Aggregation nach Gemeinden und Stadtquartieren (114 Einheiten) verglichen werden (Tab. 1). Die Variationskoeffizienten im Gürtel- und im Sektorzonenmodell sind meist nicht viel kleiner als diejenigen auf Gemeinde-/Stadtquartierebene. Wegen des Ordnungs- und Übersichtlichkeitsgewinns sind sie daher zu bevorzugen. Die geringen Prozentwerte bei der Bevölkerungsentwicklung 1920-50 beruhen auf Bevölkerungsabnahmen einiger kleiner Gemeinden - ein Blick auf die Abbildung 5 und 6 zeigt, daß die Raumgliederungen die Entwicklung durchaus prägnant darstellen können. Dank seiner feineren Aufgliederung erzielt das Sektorzonenmodell meist etwas bessere Resultate als das Gürtelmodell. Das Sektorzonenmodell ist besonders geeignet für die Darstellung von Segregationseffekten (vgl. Variablen Akademiker und Direktoren). Als Beispiel: Die Verteilung der Spezialärzte ist stärker durch Segregation geprägt als die Verteilung der Hausärzte.

Der Vergleich von Gürtel- und Sektorzonenmodell deutet darauf hin, ob Zentrifugal-/Zentripetal-Effekte oder Segregationseffekte überwiegen. Aus dieser Perspektive ergibt sich, daß die Bevölkerungsentwicklung in den Perioden 1941-60 und 1980-90 von einer sich verstärkenden Segregation begleitet war, während 1960-80 die Zentrifugalkräfte dominierten.

Selbst mit diesen einfachen Modellen sind neue, nichttriviale Erkenntnisse möglich, die aus einer Gemeindekarte nicht ohne weiteres ablesbar sind. Es wäre daher zu begrüßen, wenn dieser Artikel einen Anstoß für ähnliche Vergleiche mit anderen, eventuell räumlich nichtzusammenhängenden Regionalisierungen oder Typisierungen, anderen Variablen und anderen Testgebieten geben könnte. 

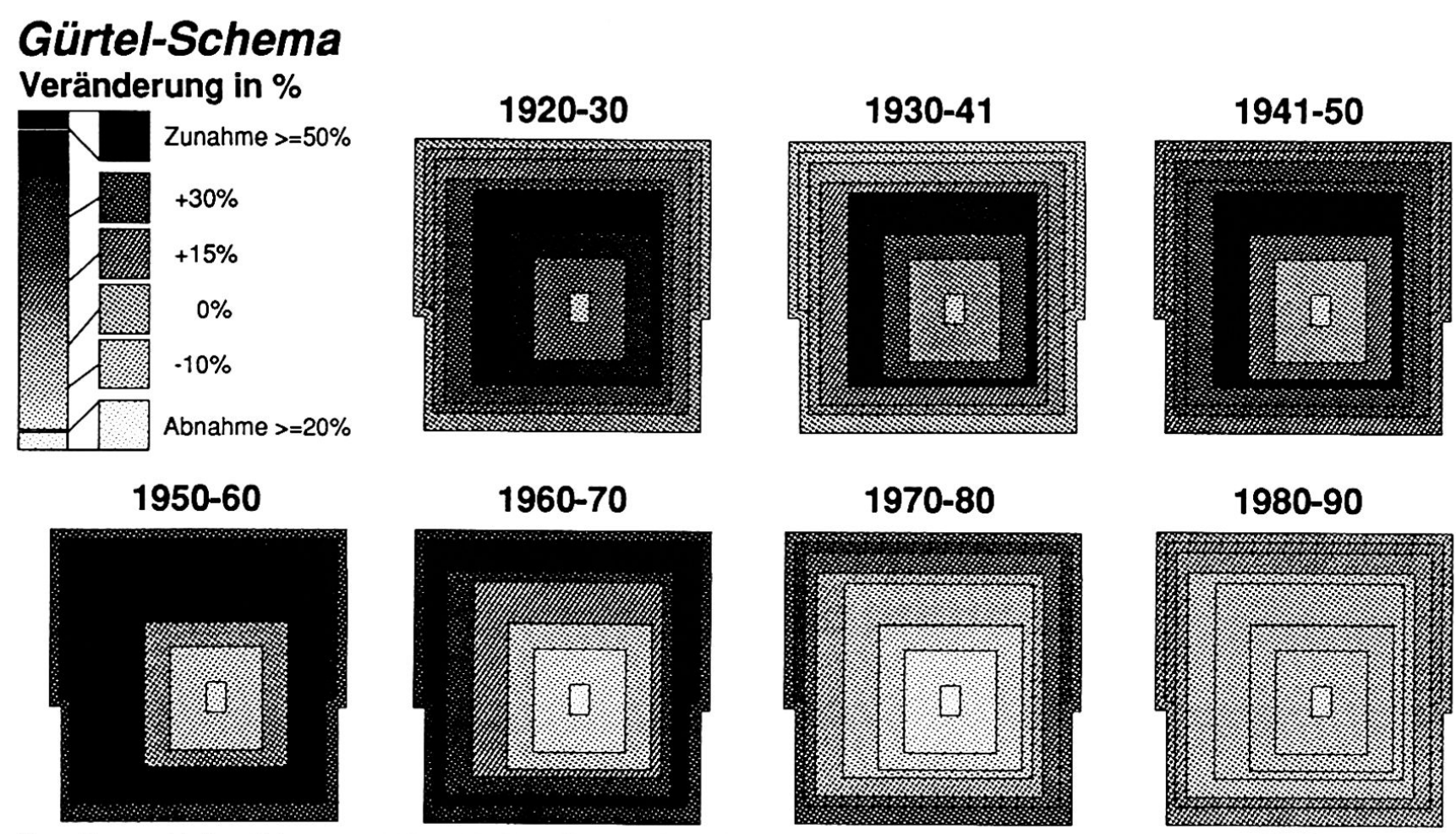

Grundlagen: Volkszăhlungen 1920-1980, Bevőlkerungsfortschreibung 1990

M. Bopp, 1991

Abb. 5 Bevölkerungsentwicklung in der Agglomeration Zürich 1920-1990 gemäß Gürtelschema (Grundlagen: Volkszählungen 1920-1980, Bevölkerungsfortschreibung 1990).

\section{Sektorzonen-Schema}

Veränderung in \%
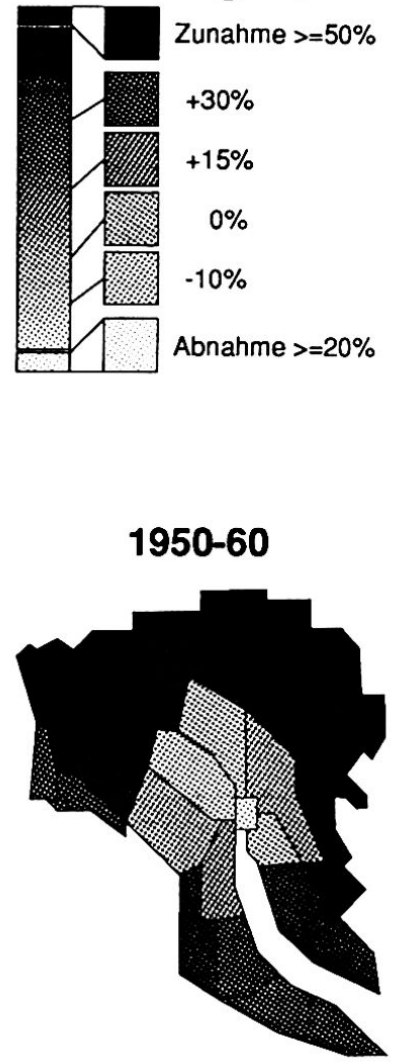

Grundlagen: Volkszăhlungen 1920-1980, Bevölkerungsfortschreibung 1990
$1930-41$

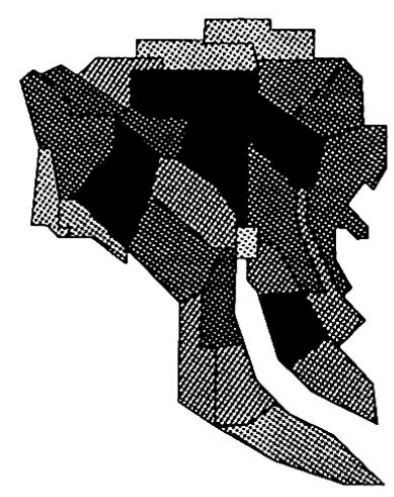

$1960-70$

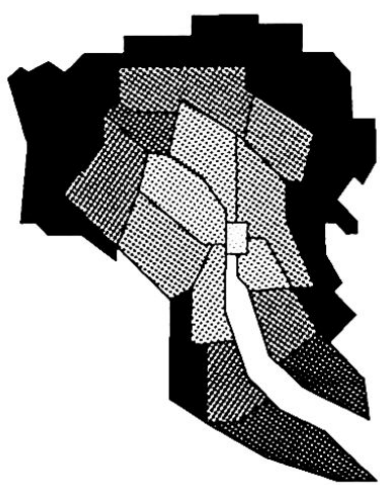

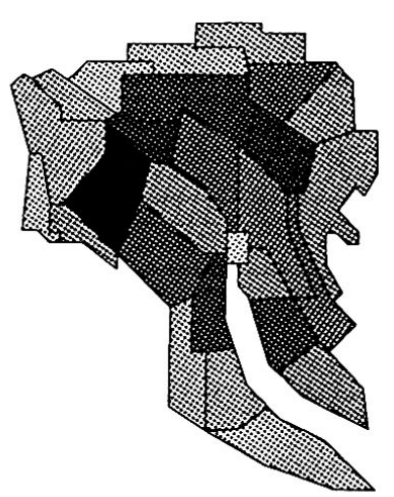

$1970-80$

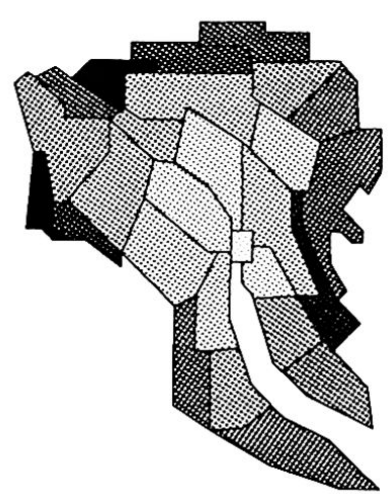

Abb. 6 Bevölkerungsentwicklung in der Agglomeration Zürich 1920-1990 gemäß Sektorzonenschema (Grundlagen: Volkszählungen 1920-1980, Bevölkerungsfortschreibung 1990). 
Tab. 1 Variationskoeffizienten im Vergleich.

\begin{tabular}{|c|c|c|c|c|}
\hline Variable (Agglomerationsmittel) & \multicolumn{2}{|c|}{$\begin{array}{l}\text { Gemeinden/Stadtquartiere } \\
\qquad n=114\end{array}$} & $\begin{array}{l}\text { Sektorzonen } \\
n=27\end{array}$ & $\begin{array}{c}\text { Gürtel } \\
n=8\end{array}$ \\
\hline $\begin{array}{l}\text { Bevölkerungsentwicklung (EZ=Einwohnerzal } \\
\text { EZ } 1930 \text { / EZ } 1920(1.202) \\
\text { EZ } 1941 \text { / EZ } 1930(1.121) \\
\text { EZ } 1950 \text { / EZ } 1941(1.167) \\
\text { EZ } 1960 \text { / EZ } 1950(1.244) \\
\text { EZ } 1970 \text { / EZ } 1960(1.168) \\
\text { EZ } 1980 \text { / EZ } 1970(1.007) \\
\text { EZ } 1990 \text { / EZ } 1980(1.019)\end{array}$ & $\begin{array}{r}74.0 \\
48.6 \\
183.9 \\
38.1 \\
54.1 \\
30.9 \\
17.9\end{array}$ & $\begin{array}{l}100 \% \\
100 \% \\
100 \% \\
100 \% \\
100 \% \\
100 \% \\
100 \%\end{array}$ & $\begin{array}{l}21.8 \% \\
28.2 \% \\
25.3 \% \\
80.5 \% \\
53.3 \% \\
69.5 \% \\
57.7 \%\end{array}$ & $\begin{array}{r}21.1 \% \\
29.3 \% \\
9.3 \% \\
67.5 \% \\
74.1 \% \\
72.8 \% \\
54.7 \%\end{array}$ \\
\hline $\begin{array}{l}\text { Bevölkerung in Privathaushalten } 1980 \\
\text { Direktoren und leitende Angestellte* }(10.9 \%) \\
\text { Akademiker }(5.7 \%)\end{array}$ & $\begin{array}{l}55.4 \\
79.4\end{array}$ & $\begin{array}{l}100 \% \\
100 \%\end{array}$ & $\begin{array}{l}92.5 \% \\
86.1 \%\end{array}$ & $\begin{array}{l}42.1 \% \\
61.7 \%\end{array}$ \\
\hline 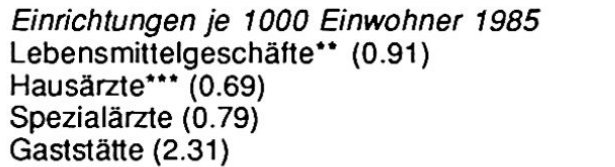 & $\begin{array}{l}100.4 \\
149.8 \\
357.3 \\
150.1\end{array}$ & $\begin{array}{l}100 \% \\
100 \% \\
100 \% \\
100 \%\end{array}$ & $\begin{array}{l}63.2 \% \\
69.8 \% \\
77.1 \% \\
95.7 \%\end{array}$ & $\begin{array}{l}85.1 \% \\
81.4 \% \\
60.4 \% \\
82.0 \%\end{array}$ \\
\hline
\end{tabular}

\section{Anmerkungen}

' Herleitung und Beschreibung finden sich in jedem Lehrbuch der Stadtgeographie (z. B. CARTER 1980 oder HERBERT 1972). Auf das mehr hierarchisch orientierte Mehr-Kerne-Modell von HARRIS und ULLMAN (1945) soll hier nicht eingegangen werden.

${ }^{2}$ Die Agglomerationsgürtel werden auch für Publikationen des Statistischen Amtes der Stadt Zürich verwendet, allerdings ohne Aufteilung der Stadt Zürich.

${ }^{3}$ Da aggregierte Werte stets eine geringere Variationsbreite aufweisen, würde dasselbe Vorgehen bei einer traditionellen Karte mit 5-8 Klassen dazu führen, daß die Aggregate in die Mittelklasse(n) fallen, wodurch der Informationsgehalt gegen Null absänke.

\section{Literatur}

BAJKA, L. (1967): Die Stadtagglomeration Zürich aus der Sicht der Planung. Dissertation Uni Zürich. Pfäffikon ZH.

BAHRENBERG, G./GIESE, E. (1975): Statistische Methoden und ihre Anwendung in der Geographie. Stuttgart.

BERTIN, J. (1974): Graphische Semiologie. Berlin/New York (frz. Original 1973).

BOPP, M./HERZOG, A. (1987): Disaggregation von Bevölkerungsdaten - Probleme und Lösungsansätze am Beispiel der Agglomeration Zürich. In: Geographica Helvetica 42, S. 211-220.

BOPP, M. (1991): Die Versorgungslage der Bevölkerung in der Agglomeration Zürich - unter besonderer Berücksichtigung des Lebensmitteldetailhandels. Dissertation Geographisches Institut der Uni Zürich (= Geo-Processing Reihe, Vol.16).

CAROL, H. (1956): Sozialräumliche Gliederung und planerische Gestaltung des Großstadtbereiches - Dargestellt am Beispiel Zürich. In: Raumforschung und Raumordnung, Band 14/2-3, S. 80-92.
CARTER, H. (1980): Einführung in die Stadtgeographie. Stuttgart (engl. Originalausgabe 1975).

GRIGG, D. (1970): Die Logik von Regionssystemen. In: BARTELS, D. (Hrsg.), Wirtschafts- und Sozialgeographie, Köln/ Berlin (= Neue wissenschaftliche Bibliothek 35, S. 183-211; engl. Original 1965: AAAG 55, p. 465-491).

GÜLLER, P./MUGGLI, Ch./SCHULER, M./STUDER, T. (1980): Entflechtungs- und Vermischungsprozesse in urbanen Räumen - Empirische Untersuchungen in den Agglomerationen Zürich, Basel und Lugano (=Arbeitsberichte NFP-5, Band 12).

HAGGETT, P. (1973): Einführung in die kultur- und sozialgeographische Regionalanalyse. Berlin/New York (engl. Originalausgabe 1965)

HAGGETT, P. (1975): Geography - a modern synthesis. New York (2nd edition).

HARVEY, D. (1979): Explanation in Geography. London (Erstausgabe 1969).

HARVEY, D. (1973): Social Justice and the City. London.

HERBERT, D. (1972): Urban Geography - a social perspective. Newton Abbot/London/Vancouver.

HERZOG, A. (1988): Desktop Mapping. In: Geographica Helvetica 43, S. $21-26$.

LEY, D. (1983) - A social Geography of the City. New York.

NEBEN, V. (1985): Die Standortstruktur des Hamburger Ladeneinzelhandels - Ein Vergleich mono- und polyzentrischer Konzeptionen. Dissertation Uni Hamburg.

PFEIL, E. (1972): Großstadtforschung - Entwicklung und gegenwärtiger Stand. Hannover (2. Auflage).

SCHULER, M. (1984): Abgrenzung der Agglomerationsräume in der Schweiz 1980 (= Beiträge zur schweizerischen Statistik, Heft 105).

SEDLACEK, P. (1978, Hrsg.): Regionalisierungsverfahren. Darmstadt (= Wege der Forschung CXCV). 